study population. Separate conclusions regarding UK specific data will be drawn. Areas for further study and recommendations for improving clinical practise will be suggested.

\section{P 036 PATIENT AND FAMILY EXPERIENCES OF DNACPR DISCUSSIONS: AN INTEGRATIVE REVIEW OF THE LITERATURE}

CC Hall, E Carduff, J Lugton, J Spiller. Marie Curie Hospice, Edinburgh

10.1136/bmjspcare-2014-000838.39

Introduction DNACPR decisions and the communication of such advance decisions have relevance in all areas of clinical care. An advance decision not to attempt CPR is taken if such an attempt is deemed be futile or if the patient does not wish it to occur. Following the recent release of revised National Guidance from the British Medical Association, the Resuscitation Council (UK) and the Royal College of Nursing, there is an increased awareness of good practice guidance around DNACPR decision making and communication. However little is known about patients' and their family members' experience of DNACPR discussions. This information will be useful in informing the development of recommendations for current clinical practise.

Aim(s) and method(s) The aim of this review is to identify patient and family views on DNACPR decisions and their experiences after having had DNACPR discussions. Methods: A literature search of multiple databases was performed including all (adult) patients in hospital, hospice and community settings, for all countries over the last 10 years.

Results Initial search results generated 558 papers. Abstracts were coded by relevance, power of study and country of origin. Exclusion criteria were applied and full texts were analysed to elucidate and summarise important themes. Analysis is currently on going. Conclusion(s) Conclusions will be drawn taking in to account limitations such as cultural differences for the 